\title{
Effects of fixation and optokinetic stimulation on vestibulo-ocular reflex suppression
}

\author{
BR CHAMBERS, MA GRESTY
}

\author{
From the MRC Neuro-Otology Unit, Institute of Neurology, National Hospital, Queen Square, London
}

SUMMARY Suppression of the vestibulo-ocular reflex was assessed in normal subjects and patients with neurological disorders to determine the relative effects on suppression of a single fixation target and an optokinetic field. Subjects were rotated sinusoidally in yaw at varying frequencies of up to $0.5 \mathrm{~Hz}$ whilst seated in a Barany chair. A comparison was made between eye movements in darkness, those produced during fixation on a central target mounted to the chair, and eye movements during fixation on the target plus an "earth-fixed" or "chair-fixed" visual background. Presentation of a background produced only minimal effects on the suppression of the vestibuloocular reflex in normal subjects. In patients with impairment of fixation suppression, suppression of the vestibulo-ocular reflex was not improved after presentation of either form of optokinetic field. The results demonstrate that central fixation is the predominant requirement for suppression of the vestibulo-ocular reflex. This correlates closely with the ability to pursue. Although the optokinetic reflex generates following eye movements similar to pursuit, it cannot be used to mediate suppression of the vestibulo-ocular reflex in the absence of an intact pursuit system. The findings strengthen the view that the optokinetic reflex evolved to act in synergy with the vestibuloocular reflex in generating compensatory eye movements.

The vestibulo-ocular reflex generates eye movements which compensate for angular displacement of the head and maintain the visual axes "on target". If one observes a moving target by turning the head, so that the target remains stationary relative to the head, then the eye movements generated by the vestibuloocular reflex are inappropriate and must be suppressed. This is termed vestibulo-ocular reflex suppression, and it is usually measured by comparison of induced nystagmus before and after presentation of a central fixation target (fixation suppression). Barnes, Benson and Prior have shown in normal humans, that when suppression of the vestibulo-ocular reflex and pursuit are compared, similar frequency response curves are obtained, with a fall off in performance at frequencies higher than 1 Hz. ${ }^{1}$ This led to the hypothesis that suppression of the vestibulo-ocular reflex depends directly on information derived from the smooth pursuit system. This was supported by the clinical observation that in patients with impaired smooth pursuit, suppression of the vestibulo-ocular reflex was abnormal. ${ }^{23}$

Address for reprint requests: Dr BR Chambers, MRC- NeuroOtology Unit, Institute of Neurology, National Hospital, Queen Square, London WC1N 3BG, UK
Quantitative analysis has shown a dissociation between pursuit and suppression of the vestibuloocular reflex in some patients with neurological disorders. ${ }^{4}$ This is most likely explained by a selective interruption of visual pathways mediating pursuit or suppression distal to the lower level of common functional and anatomical organisation.

The optokinetic reflex has probably evolved to act in synergy with the vestibulo-ocular reflex. ${ }^{56}$ At low frequencies of head rotation $(<1 \mathrm{~Hz})$, the gain of the vestibulo-ocular reflex is less than unity and the optokinetic reflex responds to slip of the image of the visual world on the retina and helps maintain the visual axes "on target", as well as providing subjective information about rotation of the head in space. ${ }^{7}$ Because the optokinetic reflex generates following eye movements similar to those of the pursuit reflex, the question arises as to whether or not the signals relating to the optokinetic reflex can be used to suppress the vestibulo-ocular reflex in a similar way to the pursuit reflex. If that were the case, then the optokinetic reflex would have an antagonistic role with respect to vestibular function, in contrast to the established synergistic relationship. The problem is difficult to resolve in normal subjects because the pursuit and the optokinetic reflex have 


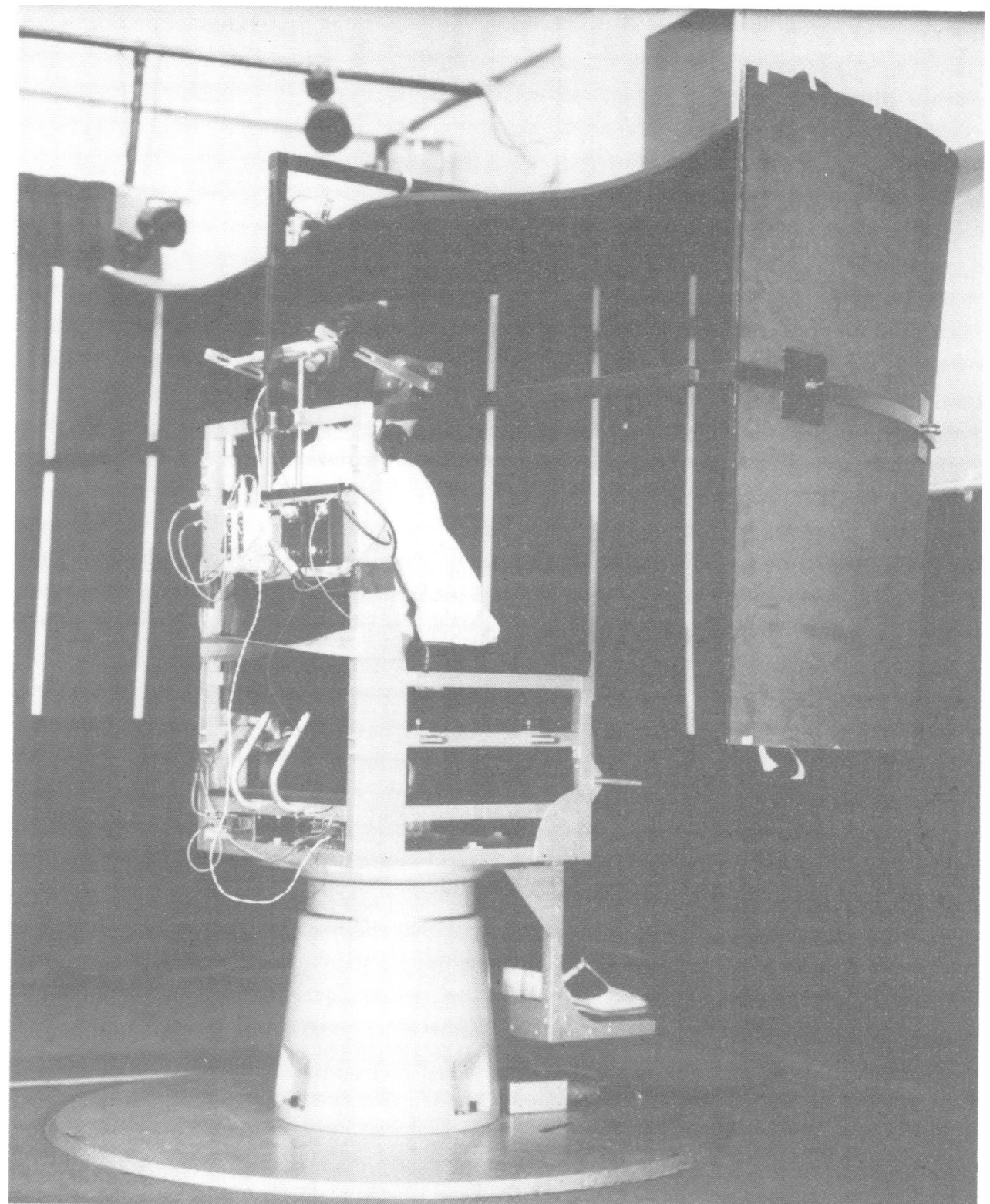

Fig 1 The chair-mounted visual perimeter which when illuminated provided an optokinetic field "sympathetic" to a central fixation target attached to the perimeter.

similar dynamic characteristics. ${ }^{8}$ However, in patients with neurological disorders, it is common to find impairment of pursuit and fixation suppression, with relatively intact optokinetic nystagmus. In these people the contribution of the optokinetic reflex to suppression of the vestibulo-ocular reflex, in the absence of intact pursuit, may be more readily studied.

\section{Patients and methods}

The following investigations were performed on normal subjects and neurological patients. Throughout all the tests described, eye movements and nystagmus were recorded using conventional DC coupled electro-oculography. Smooth pursuit in the horizontal plane was measured using a moving laser target projected onto a tangent screen two metres from the subject, by means of a mirror mounted on a 


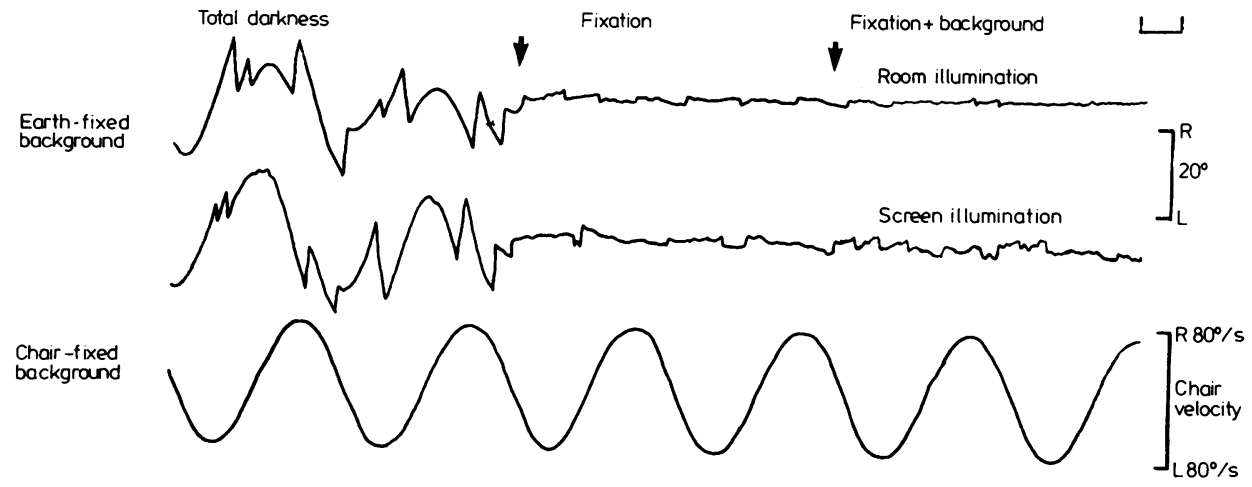

Fig 2 Raw electro-oculogram records from a normal subject illustrating suppression of vestibular responses when the subject was presented with a fixation target and then background. The upper trace was obtained when the background was provided by room illumination, and the lower trace when using the chair-mounted visual perimeter. Frequency of chair oscillation $0.4 \mathrm{~Hz}$. Peak velocity $80 \mathrm{deg} / \mathrm{s}$.

scanning motor. The target subtended an angle of less than one degree. Target motion was sinusoidal with a peak displacement of 20 degrees and the frequency was varied through the range of normal human pursuit $(0-1 \mathrm{~Hz})$. Optokinetic nystagmus was elicited using an internally illuminated motorised striped drum completely enclosing the subject, which was rotated at velocities of up to $40 \mathrm{deg} / \mathrm{s}$. Vestibulo-ocular reflex function was studied by measuring responses to sinusoidal rotation and impulsive angular acceleration and deceleration. Three tests of suppression of the vestibulo-ocular reflex were employed to assess fixation suppression alone and in combination with either an earthfixed or a chair-fixed optokinetic field. Subjects were seated and comfortably restrained in a Barany chair driven by a $17.8 \mathrm{Nm}$ torque motor. A fixation light of the same intensity and colour as the laser pursuit target, and subtending a similar visual angle, was mounted to the chair in front of the subject at a distance of one metre. The subject was rotated sinusoidally in darkness for a few seconds, and then presented with the fixation target alone. A few seconds later a stationary (that is earth-fixed) background was presented by illumination of the room, which provided contrasting velocity information. The procedure was then repeated with the subject enclosed within a chair-mounted visual perimeter, such that the entire visual environment moved with the subject ("sympathetic" field). The perimeter was a semi-circular striped screen of one metre radius, covered by a roof (fig 1). It was internally illuminated and the subject could be presented with a fixation light alone or in combination with the illuminated screen. Frequencies of oscillation were varied through the lower end of the frequency response range of the vestibulo-ocular reflex $(<0.5 \mathrm{~Hz})$. When normal subjects were tested the peak velocity of rotation was increased to a level exceeding the threshold at which suppression of the vestibulo-ocular reflex was no longer complete, whilst for the patients a peak velocity of 40 degrees/s always exceeded this threshold. The advantage of these methods for testing suppression of the vestibulo-ocular reflex was that the conditions could be changed instantaneously whilst the subject was being rotated and comparisons were not influenced by minute to minute variation in vestibular peformance which is common if patients are drowsy or fatigued. In addition observations could be based on direct inspection of raw electrooculogram records, eliminating the need for computer assisted quantitative measurements.

The protocol described above was performed on 10 normal control subjects and on 20 patients with neurological disorders. The neurological diagnoses included multiple sclerosis, idiopathic cerebellar degeneration, olivopontocerebellar degeneration, spinocerebellar degeneration, brain stem glioma, Parkinson's disease and cerebral infarction (both forebrain and hindbrain). These cases were selected on the basis of a clinically apparent abnormality of suppression of the vestibulo-ocular reflex, using the bedside test described by Halmagyi and Gresty. ${ }^{3}$ The following cases have been selected because they illustrate some of the abnormalities of pursuit and optokinetic nystagmus encountered in patients with neurological disorders. Each patient showed gross abnormalities of optokinetic nystagmus in response to movement of a small hand held striped drum but in most cases the responses to full-field stimulation illustrated below were considerably improved.

Case 1 Brain stem and cerebellar infarction. A 68-year-old woman developed cerebellar ataxia of presumed vascular origin following general anaesthesia for nephrolithotomy. Neurological signs included a supranuclear palsy of upward gaze, horizontal gaze nystagmus, central positional nystagmus, limb incoordination and gait ataxia. On attempted pursuit there were no recognisable slow following movements and full-field optokinetic stimulation failed to elicit optokinetic nystagmus (fig 3a, b).

Case 2 Brain stem glioma. A 24-year-old man presented with a subacute onset of tinnitis, unsteadiness of gait, nausea and vomiting, diplopia and hemisensory disturbance. On examination there was 1st degree nystagmus to the right, a left 6th nerve palsy, a left lower motor neuron facial weakness, left sided deafness, and a right sided sensory-motor deficit involving face, arm and leg, with marked right sided ataxia. The deficit was 
(a)

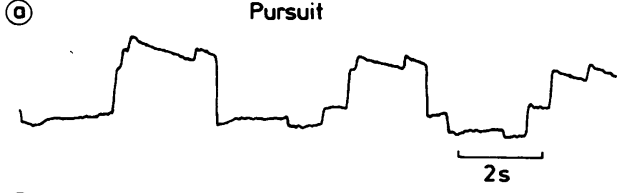

(b)

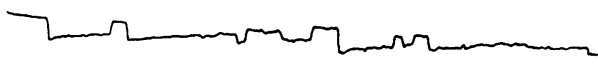

$\uparrow$ Drum R $30^{\circ}$

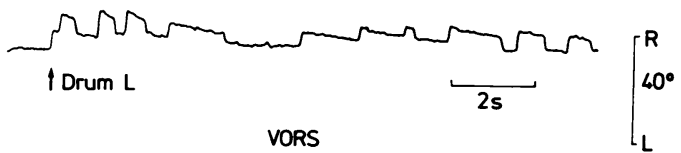

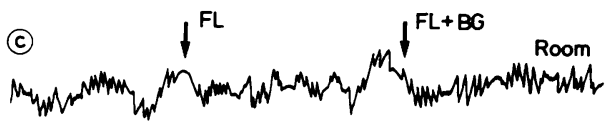

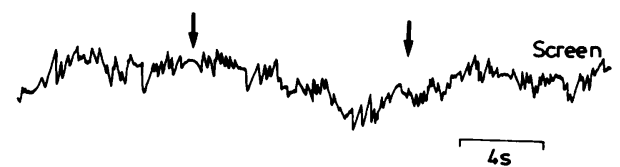

Fig 3 Case 1. Brain stem and cerebellar infarction. (a) attempted pursuit (sinusoidal target motion, frequency 0.2 $\mathrm{Hz}$, peak to peak displacement $40 \mathrm{deg}$ ). (b) responses to full-field optokinetic stimulation (constant velocity $30 \mathrm{deg} / \mathrm{s}$ ). (c) attempted suppression of the vestibulo-ocular reflex when presented with fixation target alone and with optokinetic fields (frequency $0.4 \mathrm{~Hz}$, peak velocity $40 \mathrm{deg} / \mathrm{s}$ ).

progressive and he later developed the "one and a half syndrome". A CT scan suggested the presence of a pontine glioma which was subsequently confirmed by biopsy of the lesion. When examined soon after the initial presentation, horizontal pursuit was saccadic in both directions (fig 4a). The optokinetic nystagmus gain was 0.4 with the drum rotating at $40 \mathrm{deg} / \mathrm{s}$, and with the drum moving to the right the response was irregular and poorly sustained (fig 4b).

Case 3 Idiopathic cerebellar degeneration. A 23-year-oldwoman developed a slowly progressive degenerative condition with oscillopsia, downbeat nystagmus, dysarthria, incoordination and gait ataxia. There was no family history. Structural anomalies at the level of the craniocervical junction were excluded by negative computerised tomography and myelography. Attempted following produced saccadic pursuit movements (fig 5a) and full-field optokinetic stimulation produced a poor initial response followed by a slow buildup of optokinetic nystagmus which reached a peak after about 20 seconds (fig $5 b)$.

Case 4 Right parietal infarction. A 36-year-old woman teacher was admitted after collapsing in the playground of the school. She had regained consciousness and was aware of a right frontal headache and left sided weakness. On examination there was a mild left hemiparesis and pronounced visual and sensory inattention. She became drowsy and a CT scan demonstrated infarction within the

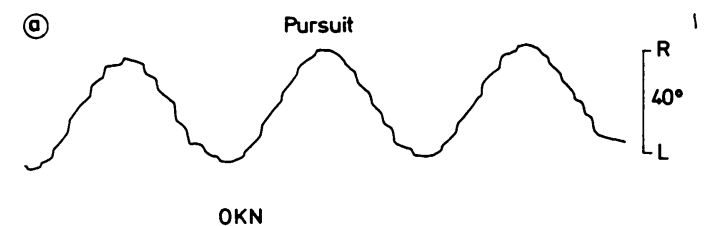

(b)
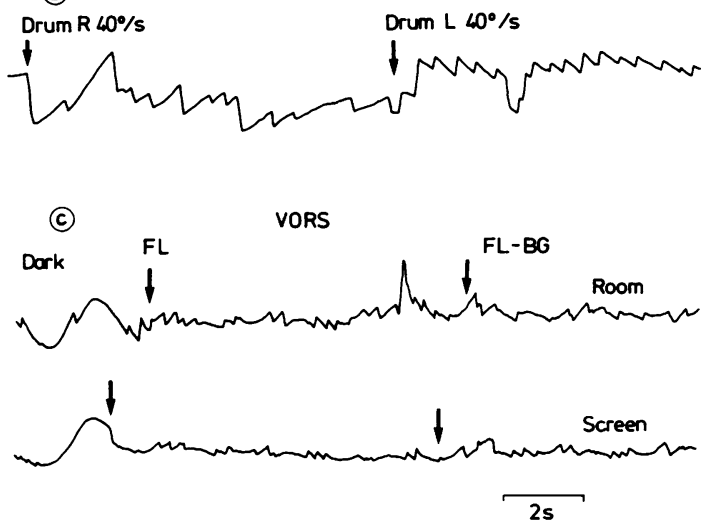

Fig 4 Case 2. Brain stem glioma. (a) attempted pursuit $(0 \cdot 2$ $\mathrm{Hz}, 40 \mathrm{deg}$ ). (b) optokinetic nystagmus generated by fullfield optokinetic stimulation $(40 \mathrm{deg} / \mathrm{s})$. (c) attempted suppression of the vestibulo-ocular reflex when presented with fixation target alone and with optokinetic fields $(0.4 \mathrm{~Hz}$, $40 \mathrm{deg} / \mathrm{s})$.

territory of the middle cerebral artery with cerebral oedema. A right carotid angiogram and an echocardiograph were normal. When examined later she was fully alert and her motor deficit had recovered although inattention persisted. Pursuit movements were broken up in both directions but particularly to the right (fig 6a). Optokinetic nystagmus induced by drum rotation to the left was normal but to the right the response was marginally reduced in velocity (fig 6b).

\section{Results}

\section{A Normal subjects}

Raw electro-oculogram records from a typical control subject (fig 2) illustrate the normal responses for the tests of suppression of the vestibulo-ocular reflex. Sinusoidal rotation in total darkness $(0 \cdot 4 \mathrm{~Hz}$, peak velocity $80 \mathrm{deg} / \mathrm{s})$ induced compensatory eye movements interspersed with nystagmus quick phases. With fixation the vestibulo-ocular reflex was largely suppressed but a low amplitude nystagmus (approximately 2 degrees) was still evident. Presentation of room illumination, which provided contrasting visual information, produced a barely discernible change in the nystagmus, which was if 


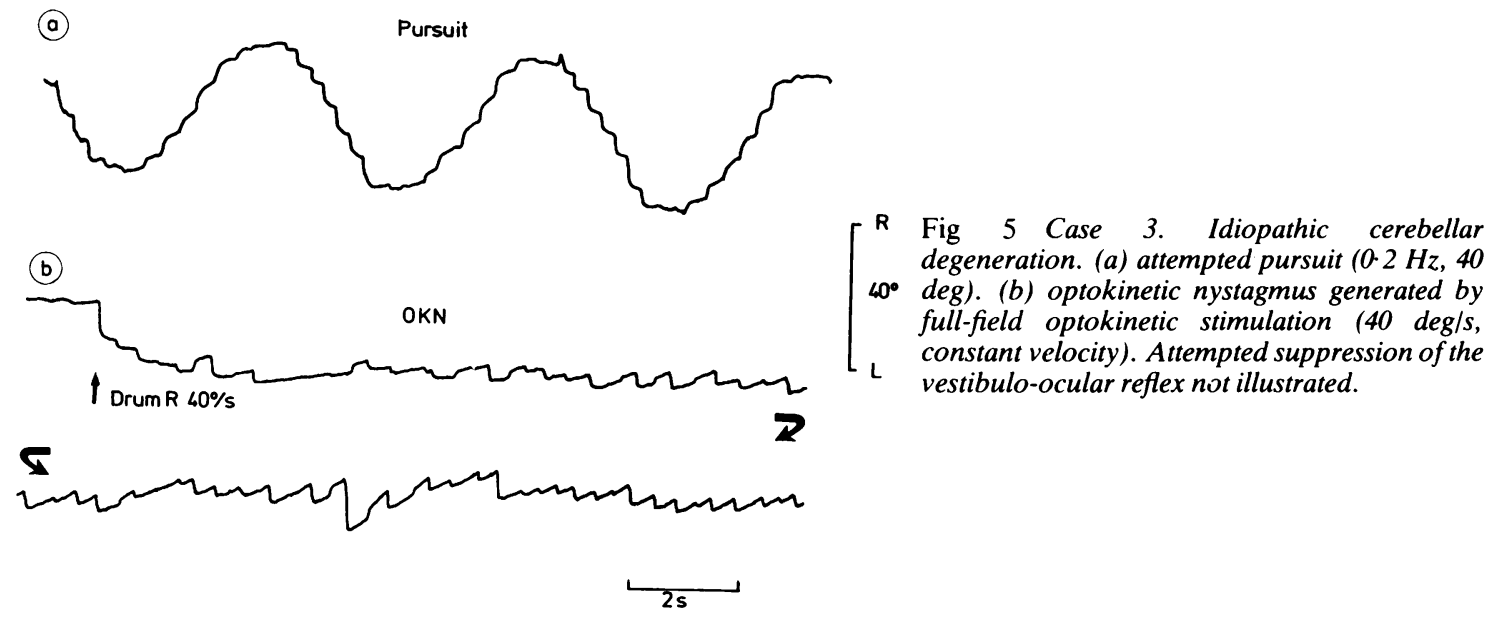

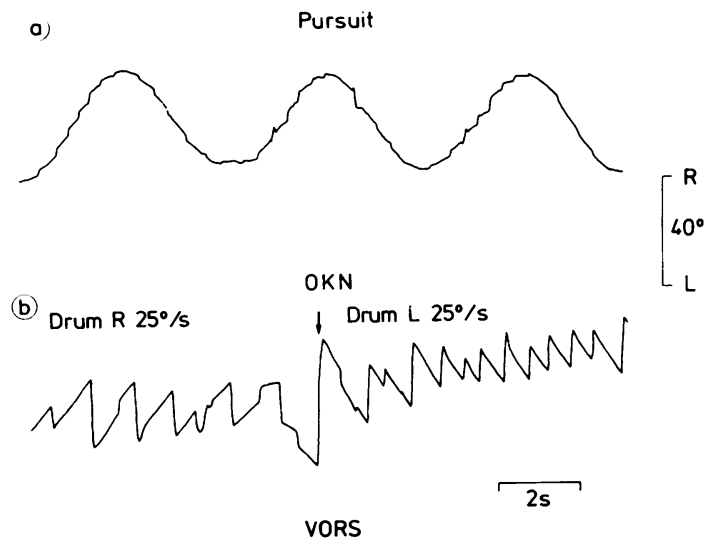

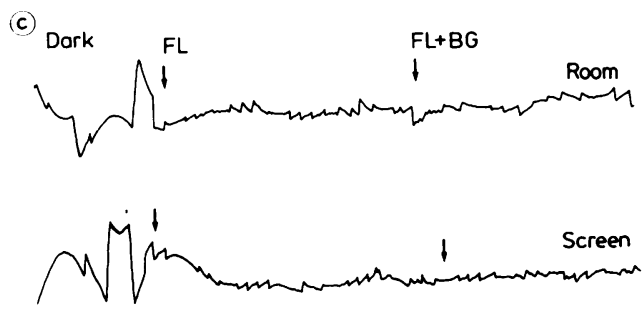

Fig 6 Case 4. Right parietal infarction. (a) attempted pursuit $\cdot 2 \mathrm{~Hz}, 40 \mathrm{deg}$ ). (b) optokinetic nystagmus generated by full-field optokinetic stimulation (25 deg/s). (c) attempted suppression of the vestibulo-ocular reflex when presented with fixation target alone and with optokinetic fields $(0.4 \mathrm{~Hz}$, $40 \mathrm{deg} / \mathrm{s})$. anything slightly better suppressed. Similarly, presentation of a "sympathetic" visual background, moving with the patient, had only a marginal effect on suppression, but in contrast the effect was adverse. These differences were consistent, but not as pronounced, for the other normal subjects. Although discernible on raw electro-oculogram records, the effects were not quantifiable because of the low amplitude of the nystagmus.

\section{B Patients}

The raw electro-oculogram records obtained from case 1 during attempted suppression of the vestibuloocular reflex (fig 3c) show that there was no fixation suppression of vestibular nystagmus, and no improvement of suppression of the vestibulo-ocular reflex after presentation of either form of optokinetic field. The records from case 2 (fig $4 c$ ) demonstrate that the patient had some fixation suppression, however nystagmus of around five degrees in amplitude and modulation of eye position were still evident. There was no convincing change in the nystagmus after presentation of either optokinetic field. Case 3 was able to centre the eyes when presented with a fixation target but unable to suppress nystagmus with or without illumination of the optokinetic fields (not illustrated), even when the frequency of oscillation was reduced to $0.03 \mathrm{~Hz}$ to allow for the time constant of the development of optokinetic nystagmus. The records of suppression of the vestibulo-ocular reflex from case 4 showed precisely the same results as for case 2 (fig $6 \mathrm{c}$ ).

\section{Discussion}

The results of this study indicate that the optokinetic reflex has no significant physiological role in the 
suppression of nystagmus induced by oscillatory rotational stimuli. In normal subjects, suppression of the vestibulo-ocular reflex was slightly enhanced when the fixation target was seen to move against a stationary background. However when the background moved with the target suppression of the vestibulo-ocular reflex was slightly impaired. These influences were trivial. Guedry et al ${ }^{9}$ made similar observations in normal subjects and referred to unpublished work by Benson and Cline. The differences are probably accounted for by an effect on pursuit. It would seem that movement of a target across a stationary background permits a more accurate assessment of the target's relative velocity; and that this information facilitates the pursuit reflex. ${ }^{10}$ The fact that the two forms of optokinetic field had opposite influences in normal subjects argues against a direct effect on the suppression mechanism.

In some patients, in whom suppression of the vestibulo-ocular reflex and pursuit were only mildly impaired, trivial effects similar to those seen in normals were observed after presentation of the optokinetic fields. However, in none of the patients was the velocity threshold at which suppression failed modified by more than a few degrees/s. This was of no functional benefit in relation to the degree of impairment of suppression of the vestibulo-ocular reflex. Many of the patients had some capacity to suppress. It is probable that amongst these there were cases in whom the mechanism mediating suppression of the vestibulo-ocular reflex was intact at the level of the flocculus (for example case 5). In general the degree of impairment of fixation suppression was proportional to the pursuit deficit, and could be attributed to a lack of input into the suppression of the vestibulo-ocular reflex mechanism of signals concerning relative target motion. In no case was it demonstrated that optokinetic information gained access to this mechanism.

In humans "passive" optokinetic nystagmus, ${ }^{11}$ induced when the subject stares at the stimulus field rather than following a particular feature, is probably derived from two components. The dominant component has a time constant of the order of $200 \mathrm{~ms}$, is dependent on cortical connections and has been referred to as a form of "involuntary" pursuit. In certain disorders this is deficient and optokinetic stimulation produces a slowly developing response with a time constant of 4 to 5 seconds (for example case 3 ), which is identical to the phylogenetically older form of optokinetic nystagmus seen in lower animals. ${ }^{12}$ With case 3 , even when the frequency of oscillation was reduced sufficiently to allow for the time constant of the development of this "primitive" form of optokinetic nystagmus, provision of an optokinetic field did not improve suppression of the vestibulo-ocular reflex. The failure of optokinetic stimulation to modify suppression in the presence of full optokinetic nystagmus (in one direction), in a patient with a bilateral deficit of suppression of the vestibulo-ocular reflex (case 4 ), is the strongest evidence that there is no mechanism by which optokinetic related signals may be used for suppression of the vestibulo-ocular reflex.

The results of this study reinforce the view that the principal function of the optokinetic reflex is to act in synergy with the vestibulo-ocular reflex in generating compensatory eye movements during head rotations, since it does not appear to be antagonistic to the vestibulo-ocular reflex. As a corollary, the results provide further evidence that the pursuit. reflex, which is important for suppression of the vestibuloocular reflex, and the optokinetic reflexes (passive form) are physiologically distinct, even though both are capable of generating smooth eye movements.

Dr. BR Chambers is supported by an Alexander Piggott Wernher Training Fellowship.

\section{References}

' Barnes GR, Benson AJ, Prior ARJ. Visual-vestibular interaction in the control of eye movement. Aviat Space Environ Med 1978;49:557-64.

${ }^{2}$ Dichgans J, Von Reutern GM, Rommelt U. Impaired suppression of vestibular nystagmus by fixation in cerebellar and noncerebellar patients. Arch Psychiatr Nervenkr 1978;226:183-99.

${ }^{3}$ Halmagyi GM, Gresty MA. Clinical signs of visualvestibular interaction. J Neurol Neurosurg Psychiatry 1979;42:934-9.

${ }^{4}$ Chambers BR, Gresty, MA. The relationship between disordered pursuit and vestibulo-ocular reflex suppression. J Neurol Neurosurg Psychiatry (in press).

${ }^{5}$ Brandt T, Dichgans J, Koenig E. Differential effects of central versus peripheral vision on egocentric and exocentric motion perception. Exp Brain Res 1973;16:476-91.

${ }^{6}$ Koenig E, Allum JHJ, Dichgans J. Visual-vestibular interaction upon nystagmus slow phase velocity in man. Acta Otolaryngol 1978;85:397-410.

' Dichgans J, Schmidt CL, Graf W. Visual input improves the speedometer function of the vestibular nuclei in the goldfish. Exp Brain Res 1973;18:319-22.

${ }^{8}$ Trinker D, Sieber J, Bartual J. Schwingungsanalyse der vestibulaer, optokinetisch und durch elektrische Reizungausgelosten Augenbewegungen beim Menschen. 1 Mitteilung: Stetige Augenbewegungen: Frequenzgange und Ortskurven. Kybernetik 1961;1:21-288.

${ }^{9}$ Guedry FE, Lentz JM, Jell RM. Visual-vestibular interactions: 1 . influence of peripheral vision on suppression of the vestibulo-ocular reflex and visual acuity. Aviat Space Environ Med 1979;50:205-11. 
${ }^{10}$ Hord JD. Observations upon the role of the peripheral retina in the execution of eye movements. $O R L$ 1975;37:65-73.

" Rademaker GGJ, Ter Braak JWG. On the central me shanisms of some optic reactions. Brain 1948;71:48-
76.

12 Yee RD, Baloh RW, Honrubia V, Lau CGY, Jenkins HA. Slow buildup of optokinetic nystagmus associated with downbeat nystagmus. Invest Ophthalmol Vis Sci 1979;18:622-9. 